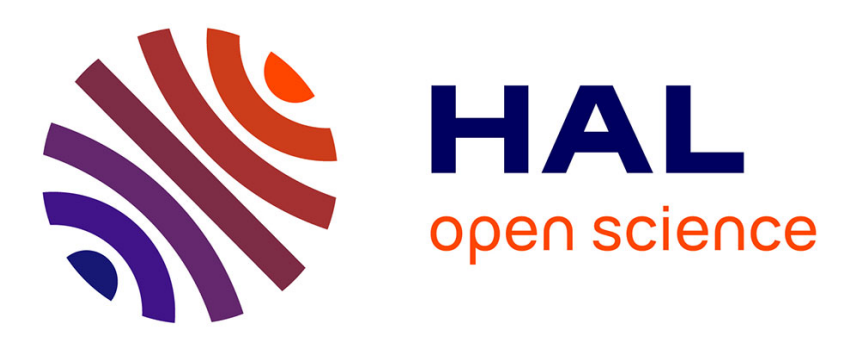

\title{
Explanation of the large spin-dependent recombination effect in semiconductors
}

D. Kaplan, I. Solomon, N.F. Mott

\section{To cite this version:}

D. Kaplan, I. Solomon, N.F. Mott. Explanation of the large spin-dependent recombination effect in semiconductors. Journal de Physique Lettres, 1978, 39 (4), pp.51-54. 10.1051/jphyslet:0197800390405100 . jpa-00231440

\section{HAL Id: jpa-00231440 https://hal.science/jpa-00231440}

Submitted on 1 Jan 1978

HAL is a multi-disciplinary open access archive for the deposit and dissemination of scientific research documents, whether they are published or not. The documents may come from teaching and research institutions in France or abroad, or from public or private research centers.
L'archive ouverte pluridisciplinaire HAL, est destinée au dépôt et à la diffusion de documents scientifiques de niveau recherche, publiés ou non, émanant des établissements d'enseignement et de recherche français ou étrangers, des laboratoires publics ou privés. 


\title{
EXPLANATION OF THE LARGE SPIN-DEPENDENT RECOMBINATION EFFECT IN SEMICONDUCTORS
}

\author{
D. KAPLAN \\ L.C.R., Thomson-C.S.F., 91401 Orsay, France \\ I. SOLOMON \\ Ecole Polytechnique, Laboratoire de Physique de la Matière Condensée, 91128 Palaiseau, France \\ and \\ N. F. MOTT \\ Cavendish Laboratory, Cambridge, England \\ (Reçu le 5 décembre 1977, accepté le 4 janvier 1978)
}

\begin{abstract}
Résumé. - Les théories précédentes concernant la variation de photoconductivité à la résonance de spin (expériences de Lépine dans le silicium) prédisent un effet de 10 à 100 fois trop faible.

Nous proposons un modèle où, par suite du temps de vie plus court d'une paire électron-trou en configuration singulet, la distribution des spins des paires contient un surplus d'états triplets. Ce surplus est supprimé par saturation de la résonance, ce qui raccourcit ainsi le temps de recombinaison.

La variation peut atteindre $10 \%$ et l'effet ne dépend pas du champ appliqué, en accord avec l'expérience.

Abstract. - Previous theories to explain the variation of photoconductivity upon saturation of electron spin resonance, as observed by Lépine in silicon, predict an effect 10 to 100 times smaller than experiment.

In the present model we show that, due to the shorter lifetime of electron-hole pairs in singlet configuration, the steady state spin distribution shows a surplus of triplet pairs. Saturation of resonance restores the random distribution, resulting in a shortening of the recombination time.

The relative variation can be as large as $10 \%$, and is field independent as confirmed by experiment.
\end{abstract}

This letter describes a model which explains spindependent recombination effects in semiconductors [1]. By this we mean the type of experiments whereby one saturates an electron spin resonance (ESR) and observes a modification of the carrier recombination rate. Typically one measures photoconductivity in a sample placed in an ESR microwave cavity with an applied dc magnetic field $H_{0}$. When the magnetic field is swept through resonance a decrease of photoconductivity is observed which can be related to an increase in recombination rate. Various experiments of this kind have been reported in the case of silicon since the pioneering work of Lépine et al. [2], notably for surface recombination [3, 4], recombination in plastically deformed crystals $[5,6]$ and more recently for amorphous films grown by silane decomposition [7]. A similar effect has been observed on the current of a p-n junction which depends on the recombination rate in the space charge region of the diode [8].

An understanding of this phenomenon can start from the implications of spin angular momentum conservation : if the spin-orbit interaction is not large, as is the case for silicon, recombination transitions will conserve total spin. If after the transition the system is without a spin (as for instance for free electron-free hole recombination) then the initial configuration must be $S=0$ (singlet configuration) for the transition to take place. This consideration can be generalized to processes involving recombi- 
nation centers, in which one or both of the carriers are trapped, provided the final state is an $S=0$ state. A microwave field, strong enough to saturate the ESR of either the electron or hole, will produce an isotropic spin distribution in which the probabilities of singlet and triplet configurations will be $\frac{1}{4}$ and $\frac{3}{4}$ respectively. This will produce a change in recombination rate if the initial distribution was not isotropic. The center of the problem is the origin of this departure from isotropy.

The applied magnetic field $H_{0}$ polarizes the spins and so is responsible for some anisotropy. To calculate this one can write the projection operators $\pi_{\mathrm{T}}$ and $\pi_{\mathrm{S}}$ on the singlet and triplet manifolds as :

$$
\begin{aligned}
& \pi_{\mathrm{T}}=\frac{3}{4}+\mathbf{S}_{\mathrm{e}} \cdot \mathbf{S}_{\mathrm{h}} \\
& \pi_{\mathrm{S}}=\frac{1}{4}-S_{\mathrm{e}} \cdot \mathbf{S}_{\mathrm{h}}
\end{aligned}
$$

where $\mathbf{S}_{\mathrm{e}}$ and $\mathbf{S}_{\mathrm{h}}$ are the electron and hole spin operators. Averaging over equilibrium spin populations one obtains a probability for the singlet configuration :

$$
P_{\mathrm{S}}=\frac{1}{4}\left(1-p_{\mathrm{e}} p_{\mathrm{h}}\right)
$$

where $p_{\mathrm{e}}$ and $p_{\mathrm{h}}$ are the equilibrium spin polarizations in the applied magnetic field. This was the model first proposed by Lépine [3]. From these formulae it is apparent that the maximum change $\Delta R$ of the recombination rate $R$ is $\Delta R / R=p_{\mathrm{e}} \cdot p_{\mathrm{h}}$.

This is of order $10^{-6}$ at room temperature in a field $H_{0}=3000 \mathrm{G}$. However some experiments performed under these conditions $[3,6-8]$ have yielded changes $\Delta I_{p}$ in the photoconductivity current as large as :

$$
\frac{\Delta I_{\mathrm{p}}}{I_{\mathrm{p}}} \simeq 10^{-4}
$$

Several attempts have been made to resolve this discrepancy : $a$ ) by considering situations where $\Delta I_{\mathrm{p}} / I_{\mathrm{p}}=\alpha \Delta R / R$ with $\alpha$ larger than unity [3] ; b) by assuming a spin larger than $\frac{1}{2}$ due to ferromagnetic exchange interactions [6] ; c) by a model of resonant heating of the spins system which can also produce a change of the recombination rate proportional to the product $p_{\mathrm{e}} \cdot p_{\mathrm{h}}$ [9]. We conclude however that the magnetic field polarization falls short of explaining the magnitude of the effect in the general case.

We present here a model in which the departure from isotropy does not have its origin in the applied magnetic field. The new ingredient in this model is the concept of a pair : we assume that before recombination takes place the electron and hole go through an intermediate state in which they are in a situation of proximity. From this pair state the electron-hole system can either recombine or dissociate, but not recombine with other electrons and holes.

Examples of such recombination involving pairs are exciton recombination and donor-acceptor recombination [10]. More generally if both the electron and the hole are trapped before recombining, as for instance in highly disordered systems such as amorphous semiconductors. the concept of a pair will in many cases be approximately applicable : the recombination of a trapped electron with the nearest neighbour trapped hole will have a higher probability than with holes further away.

To understand how the existence of pairs introduces a spin correlation effect, we take the extreme case where pairs are created with random spin orientations and no spin relaxation occurs in the pair state. If this is the case, pairs created in singlet configurations have a shorter lifetime than pairs created in triplet configurations, consequently their steady state population will be smaller than in the random situation and we are left with a net surplus of triplet configurations. If one saturates the electron or hole spin resonance one returns to a random spin distribution which contains more singlets so that the recombination rate is enhanced. The pair concept is central because the spin orientation of the system as a whole remains random and it is only the orientation of the two spins in the same pair that is correlated.

To make an explicit calculation of the effect one must consider all spin configurations between the singlet and the triplet extremes. We use the usual vector representation in which to each spin quantum state (electron or hole) one associates a spin vector of length $\frac{1}{2}$ (in $\hbar$ units) which has the property that its projections on axes $x, y, z$ are equal to the quantum averages of $S_{x}, S_{y}, S_{z}$. In this representation one obtains the quantum average :

$$
\left\langle\mathbf{S}_{\mathrm{e}} \cdot \mathbf{S}_{\mathrm{h}}\right\rangle=\frac{\cos \theta}{4}
$$

where $\theta$ is the angle between electron and hole spin vectors and is the only parameter necessary to characterize the spin distribution for our purpose. For such a pair, the recombination probability per unit time can be expressed as

$$
W_{\mathbf{R}}(\theta)=W_{\mathbf{S}}\left\langle\pi_{\mathbf{S}}(\theta)\right\rangle
$$

where $W_{\mathrm{S}}$ is the recombination rate for a pair in a pure singlet configuration. This assumes, for simplicity, that the triplet recombination is completely forbidden.

Using (1), (3) and (4), one obtains :

$$
W_{\mathbf{R}}(\theta) \doteq W_{\mathrm{S}} \frac{1-\cos \theta}{4} .
$$

If the pairs with an angle between $\theta$ and $\theta+\mathrm{d} \theta$ are created at the rate $c(\theta)$ and dissociate at a rate $W_{\mathrm{D}}$ [11], the steady state density of pairs with angle $\theta$ is given by detailed balance : '

$$
N(\theta)=\frac{c(\theta)}{W_{\mathbf{R}}(\theta)+W_{\mathrm{D}}} .
$$


In general $c(\theta)$, appearing in the usual rate equation [12], will depend on hole concentration $p$, electron concentration $n$ and possibly on pair concentration $N(\theta)$. For simplicity and illustration purposes, we shall assume that the possible pair sites are weakly populated (which is certainly the case for an amorphous semiconductor with a large number of states in the gap) so that the creation rate is independent of the steady state concentration of the pair density $N(\theta)$. The pairs being created by capture from carriers with an isotropic spin distribution, it is reasonable to assume an isotropic distribution for $c(\theta)$ which then takes the form :

$$
c(\theta)=\frac{C}{2} \sin \theta
$$

where $C$ is the total creation rate depending only on the concentrations $n, p$ of injected carriers.

The variation of the density of pairs with angle $\theta$ takes the form :

$$
N(\theta)=\frac{2 C \sin \theta}{4 W_{\mathrm{D}}+W_{\mathbf{S}}(1-\cos \theta)} .
$$

For comparison, we consider the case where the recombination rate $W_{\mathrm{R}}$ would be equal to the average value $W_{\mathrm{S}} / 4$, independent of the spin orientations. We then get the random distribution :

$$
N_{\mathrm{RAND}}(\theta)=\frac{2 C \sin \theta}{4 W_{\mathrm{D}}+W_{\mathrm{S}}} .
$$

Figure 1 shows a plot of $N(\theta) / N_{\text {RAND }}(\theta)$ for various values of the ratio $\lambda=W_{\mathrm{D}} / W_{\mathrm{S}}$. As expected, when the dissociation $W_{\mathrm{D}}$ is not too fast compared to the

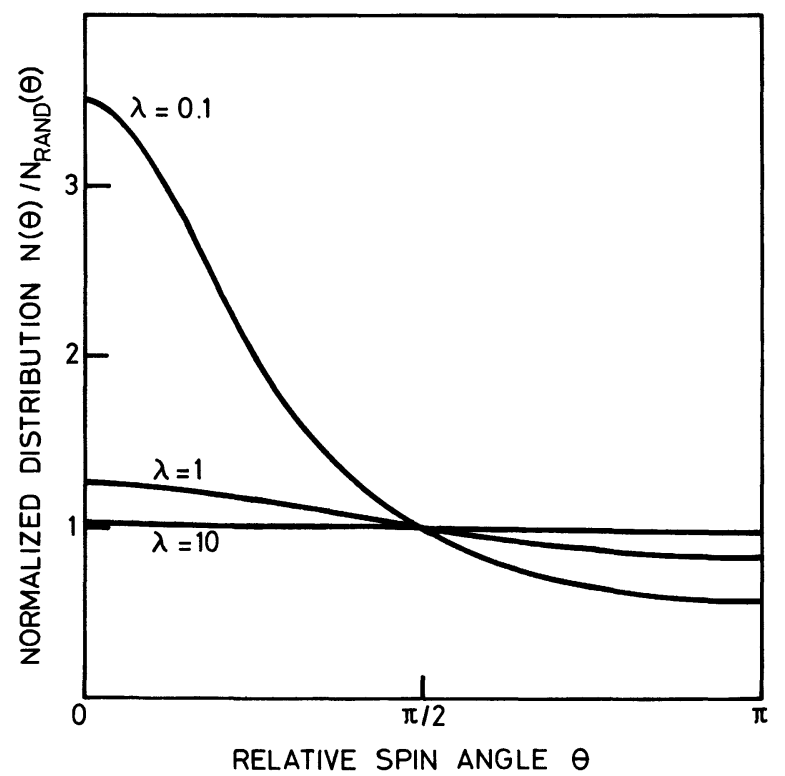

FIG. 1. - Steady state distribution of the angle $\theta$ between spins of the electron-hole pairs compared to a random distribution (see text), for different values of $\lambda=W_{\mathrm{D}} / W_{\mathrm{S}}$

$$
\frac{N(\theta)}{N_{\mathrm{RAND}}(\theta)}=\frac{1+4 \lambda}{1+4 \lambda-\cos \theta} \text {. }
$$

singlet recombination rate $W_{\mathrm{S}}$ (i.e. $\lambda$ not too large) the distribution is peaked at $\theta=0$, showing a surplus of triplet orientations.

With this distribution, we are able now to calculate the magnitude of the spin-dependent effect on recombination. The total recombination rate is obtained by summing $N(\theta) W_{\mathbf{R}}(\theta)$ over all possible values of $\theta$ :

$$
R=\int_{0}^{\pi} N(\theta) W_{\mathrm{R}}(\theta) \mathrm{d} \theta .
$$

Using (5) and (8) this gives, expressed as a function of $\lambda=W_{\mathrm{D}} / W_{\mathrm{S}}$ :

$$
R=C\left[1-2 \lambda \log \frac{1+2 \lambda}{2 \lambda}\right]
$$

Now the resonant saturation of either the electron or the hole spin [13]. extrapolated to infinite microwave power, restores the spin-pair random distribution, and the pairs recombine at a rate given by the average of expression (5) :

$$
\bar{W}_{\mathrm{R}}=W_{\mathrm{S}} / 4 \text {. }
$$

The recombination rate is then simply

$$
R_{\mathrm{SAT}}=\frac{C}{1+4 \lambda} \text {. }
$$

Thus in the present model the spin dependent recombination effect is simply due to the change of the recombination rate from $R$ to $R_{\mathrm{SAT}}$ when one saturates the spin resonance line. The relative change

$$
\frac{\Delta R}{R}=\frac{R-R_{\mathrm{SAT}}}{R}
$$

is plotted in figure 2 as a function of $\lambda=W_{\mathrm{D}} / W_{\mathrm{S}}$.

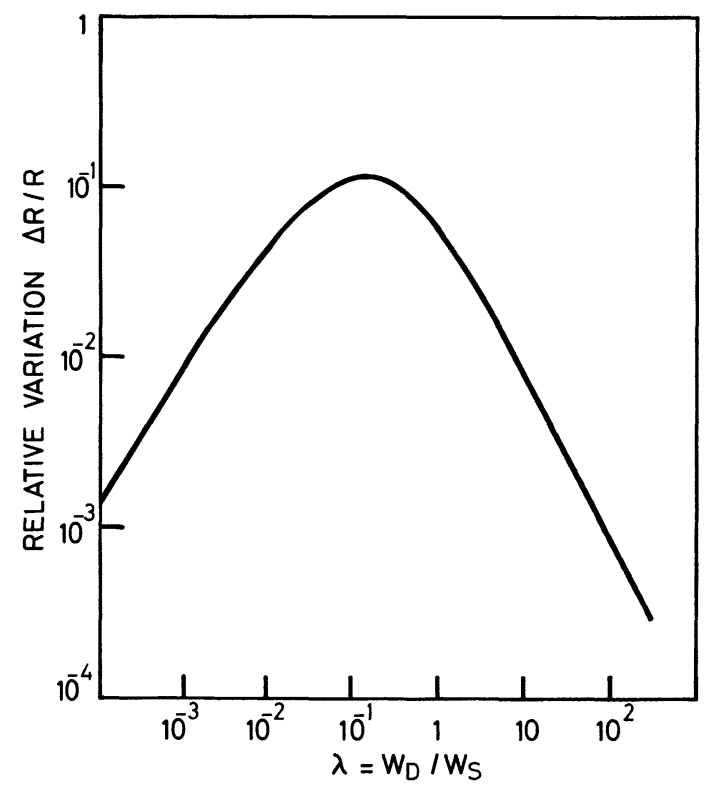

FIG. 2. - Relative variation of the recombination rate $\Delta R / R$ obtained by saturation of the ESR as a function of dissociation to singlet recombination rate ratio $\lambda=W_{\mathrm{D}} / W_{\mathrm{S}}$. For $\lambda$ varying over 6 orders of magnitude, $\Delta R / R$ remains larger than $10^{-3}$. 
A maximum effect of the order of $10 \%$ is obtained for $\lambda=0.3$. For smaller values of $\lambda=W_{\mathrm{D}} / W_{\mathrm{S}}$ the dissociation probability is small so that the recombination rate is essentially equal to the pair creation rate $C$, i.e. spin independent. For large $\lambda$ the pair lifetime is dominated by the dissociation process and the triplet enhancement in eq. (8) becomes small.

We have thus shown the possibility of relative variations of the recombination rate as high as $10 \%$ upon application of a resonant microwave field. The actual effect will in fact be smaller because of a number of considerations omitted in the present simplified picture :

- Finite triplet recombination;

- Distribution in $\lambda$ values;

- Spin relaxation processes in the pair state;

- Incomplete saturation of the resonance;

- Existence of competing recombination mechanisms that do not involve pairs.

Work is in progress to introduce these effects in the general description. Altogether they can lead to the experimentally observed value $\Delta R / R \lesssim 10^{-4}$.

Another omitted problem is the exchange interaction between an electron and a hole in a pair. If it is large compared to the difference of their Zeeman energies, the rf field will not change the angle $\theta$. This implies that the overlap between the two wavefunctions must be small, but still large enough to allow a finite recombination probability. For carriers trapped in localized states of sufficient depth, pairs having these properties can certainly exist.

The situation is different if one or both carriers are in shallow states. In this case, the Coulomb interaction may play a role, and a bound pair (exciton) can exist with large overlap, as envisaged by Pai and Enck [14] for chalcogenides and Engemann and Fischer [15] for silicon. If so, and if the mean free path is small, we may apply the classical analysis of Onsager [16] : thermal energy can separate the carriers from time to time up to a distance $e^{2} / k k T$ without them subsequently becoming free. At this distance the exchange may be small enough to allow the rf field to change $\theta$ and thus increase the probability of recombination when the carriers return to the ground state of the pair.

A remarkable feature of the present theory is that it predicts a spin-dependent effect independent of the applied magnetic field in the first order of approximation. This has been verified in a preliminary experiment [17] where the spin-dependent photoconductivity has been measured on the same amorphous silicon sample at two resonance frequencies of $9.3 \mathrm{GHz}$ and $1.9 \mathrm{GHz}$. Instead of a spin dependent effect varying like the square of the resonance field (a factor $(9.3 / 1.9)^{2}=24$ in this experiment) as predicted by the previous models $[3,6,9]$, the same variation $\Delta R / R \simeq 0.5 \times 10^{-4}$ (extrapolated to complete saturation) has been obtained at the two frequencies, thus providing a crucial test for the present theory.

\section{References}

[1] A review, by

Solomon, I., Of the spin-dependent effects in semiconductors can be found in Proc. 11th Int. Conf. Phys. of Semicond. (Polish Scientific Publishers) Warsaw (1972), p. 27.

[2] Lepine, D. and Prejean, J. J., Proc. 10th Int. Conf. Phys. of Semicond., Boston (1970).

[3] Lepine, D., Phys. Rev. B 6 (1972) 436.

[4] Ruzyho, J., Shiota, I., Miyamota, N. and Nishizawa, J., J. Electrochem. Soc. 123 (1976) 26.

[5] Wosinski, T. and Figielski, T., Phys. Status Solidi B 71 (1975) 1273

[6] Lepine, D., Grazhulis, V. A. and Kaplan, D., Proc. 13th Int. Conf. Phys. of Semicond., Roma (1976).

[7] Solomon, I., Biegelsen, D. and Knights, J. C., Solid State Commun. 22 (1977) 505.

[8] Solomon, I., Solid State Commun. 20 (1976) 215.

[9] White, R. M. and GouYet, J. F., Phys. Rev. B (to be published, November 15, 1977).

[10] Bruwin, R. F., Cavenett, B. G., Davies, J. J. and Nicholls, J. E., Solid State Commun. 18 (1976) 1283.

For a review of optical detection of spin dependent radiative recombination in semiconductors, see
Cavenett, B. C., Luminescence Spectroscopy. Edited by Lumb, M. D. (Academic Press) (in press) chap. 6.

[11] The dissociation rate $W_{\mathrm{D}}$, due to the motion of the carriers (for example, re-excitation of the electron in the conduction-band) is assumed here to be independent of $\theta$.

[12] Shockley, W. and Read, W. T., Phys. Rev. 87 (1952) 835.

[13] If the $g$-values of electrons and holes are different, the resonant transitions do not conserve the total spin of a pair, thus destroying the correlation between spin pairs. If the $g$-values are the same, the random distribution of spin states is obtained through the resonant heating of the spin system by the microwave field. See, for example,

Goldman, M., Spin Temperature and Nuclear Resonance in Solids (Clarendon Press, Oxford) 1970.

[14] PaI, D. M. and ENCK, R. C., Phys. Rev. B 11 (1975) 5163.

[15] Engemann, D. and Fischer, R., Procs 5th Int. Conf. on Amorphous and Liquid Semiconductors, Garmisch (1974) p. 1042.

[16] Onsager, L., Phys. Rev. 54 (1938) 554.

[17] Solomon, I., to be published. 